\title{
Density of states of OLED hosts from thermally stimulated luminescence
}

Andrei Stankevych, ${ }^{1}$ Alexander Vakhnin,, ${ }^{1}$ Denis Andrienko, ${ }^{2}$ Leanne Paterson, ${ }^{2}$ Jan Genoe, ${ }^{3}$ Ivan I. Fishchuk, ${ }^{4}$ Heinz Bässler, ${ }^{5}$ Anna Köhler, ${ }^{5,6}$ Andrey Kadashchuk ${ }^{1,3,{ }^{*}}$

${ }^{1}$ Institute of Physics, Natl. Academy of Sciences of Ukraine, Prospect Nauky 46, 03028 Kyiv, Ukraine

${ }^{2}$ Max Planck Institute for Polymer Research, Ackermannweg 10, 5528 Mainz, Germany

${ }^{3}$ IMEC, Kapeldreef 75, B-3001 Leuven, Belgium

${ }^{4}$ Institute for Nuclear Research, Natl. Academy of Sci. of Ukraine, Prospect Nauky 47, 03028 Kyiv, Ukraine

${ }^{5}$ Bayreuth Institute of Macromolecular Research (BIMF), Universitätsstr. 30, 95448 Bayreuth, Germany

${ }^{6}$ Soft Matter Optoelectronics and Bavarian Polymer Institute (BPS), Universitätsstr. 30, 95448 Bayreuth, Germany

\begin{abstract}
The electronic density-of-states (DOS) plays a central role in controlling the charge-carrier transport in amorphous organic semiconductors, while its accurate determination is still a challenging task. We have applied the low-temperature fractional thermally stimulated luminescence (TSL) technique to determine the DOS of pristine amorphous films of OLED host materials. The DOS width is determined for two series of hosts, namely, (i) carbazole-biphenyl (CBP) derivatives: $C B P, \mathrm{mCBP}$, and $\mathrm{mCBP}-\mathrm{CN}$, and (ii) carbazolephenyl (CP) derivatives: $\mathrm{mCP}$ and $\mathrm{mCP}-\mathrm{CN}$. TSL originates from radiative recombination of chargecarriers thermally released from the lower energy part of the intrinsic DOS that causes charge trapping at very low temperatures. We find that the intrinsic DOS can be approximated by a Gaussian distribution, with a deep exponential tail accompanying this distribution in CBP and mCBP films. The DOS profile broadens with increasing molecular dipole moments, varying from 0 to 6 Debye, in a similar manner within each series, in line with the dipolar disorder model. The same molecular dipole moment, however, leads to a broader DOS of CP compared to CBP derivatives. Using computer simulations, we attribute the difference between the series to a smaller polarizability of cations in CP-derivatives, leading to weaker screening of the electrostatic disorder by induction. These results demonstrate that the low-temperature TSL technique can be used as an efficient experimental tool for probing the DOS in small-molecule OLED materials.
\end{abstract}

* Corresponding author: kadash@iop.kiev.ua 


\section{Introduction}

The determination of the electronic density of states (DOS) in disordered organic solids is of fundamental importance for the accurate physical understanding and modeling of the charge-carrier transport in organic functional semiconductors and electronic devices. A Gaussian shape, $g(\epsilon) \propto$ $\exp \left[-1 / 2(\epsilon / \sigma)^{2}\right]$, where $\sigma$ is the width of the DOS, is a common approximation used for neat disordered organic solids [1-3], such as low-molecular-weight glasses and semiconducting polymers. Nondispersive photocurrent transients observed in amorphous organic materials [3] unambiguously support the notion of a Gaussian-shape, rather than an exponential DOS $[4,5]$. The point is that in the case of an exponential DOS, the time-of-flight (ToF) transients should always be dispersive because they would never equilibrate during the transit time at small carrier concentrations and should follow dispersive transport model. The Gaussian DOS is also predicted by the central limit theorem, since the interaction energy of a charged molecule embedded in an random polarizable environment, depends on a large number of neighboring neutral molecules $[1,2]$.

Despite the success of the Gaussian disorder formalism, particularly for neat organic semiconductor films, the exact shape of the DOS can deviate from a Gaussian shape. For instance, in the far tail region of the DOS [6] or in doped organic films where the DOS is distorted by ionized dopants [7]. Consequently, charge transport characteristics in sandwich-type diodes are often modeled by assuming that the DOS is a superposition of Gaussian and exponentially distributed trap states [8]. Recently, May et al. [9] showed that deep exponential tails can develop for molecules with large changes in molecular polarizability upon charging. Several direct experimental measurements of the DOS shape also demonstrated that the center of the DOS distribution is indeed Gaussian, but the tails can have a more complex structure $[10,11]$.

The experimental determination of the DOS profile, however, is far from trivial. In contrast to inorganic semiconductors, the DOS of organic systems is not amenable to optical probing, because the direct optical transitions between the valence and conduction states are weak and masked by strong exciton transitions $[12,13]$. A number of experimental techniques have been used to probe the DOS of organic films, such as temperature-dependent space-charge-limited-current spectroscopy [14], ultraviolet photoelectron spectroscopy (UPS) [15], inverse photoemission spectroscopy (IPS) [16], Kelvin probe force microscopy (KPFM) [10,11], electrochemically gated transistor approach [17], as well as thermally stimulated luminescence (TSL) [18]. A clear advantage of TSL is that it is a purely optical and electrodefree technique. This helps to eliminate interface/contact effects, and it allows DOS measurements in materials with low charge carrier mobilities and in systems with large energy disorder where the charge transport is very dispersive. 
TSL is the phenomenon of luminescent emission after removal of excitation (UV light in our case) under conditions of increasing temperature. First, photogenerated charge carriers populate trap states, usually at very low temperatures. Once the sample is heated up, typically with a linear temperature ramp, trapped charge carriers are released and then recombine producing luminescence emission. Technically, TSL is a relatively simple technique with a straightforward data analysis. However, it should be noted that mechanism of TSL in amorphous organic semiconductors with a broad energy distribution of strongly localized states, differs from the mechanism commonly accepted for crystalline materials with band-type transport where discrete trapping level model is applicable. A specific feature of amorphous solids is that the localized states within the lower energy part of the intrinsic DOS distribution can give rise to shallow charge trapping at very low temperatures, and as a consequence, TSL can be observed even in materials where the "trap-free limit" has been postulated. Since TSL measurements are normally performed after a long dwell time after photoexcitation, the initial energy distribution of localized carriers is formed after low-temperature energy relaxation of photogenerated carriers within a Gaussian distribution of DOS and, therefore, the first thermally assisted jumps of relaxed carriers are considered as the rate-limiting steps determining TSL. This is in contrast to thermally stimulated conductivity which is believed to be governed by the interplay between the trapping and detrapping processes [4]. Theoretical background for application of TSL for probing the DOS distribution in disordered organic systems has been developed $[18,19]$ using a variable-range hopping formalism and the concept of thermally stimulated carrier random walk within a positionally and energetically random system of hopping sites. The theory proves that the high temperature wing of the TSL curve in such amorphous materials should be an exact replica of the deeper portion of the DOS distribution $[18,19]$ and yields the effective DOS width.

TSL is particularly suitable for probing the DOS and extrinsic traps in luminescent organic materials, such as $\pi$ - and $\sigma$-conjugated polymers[18,20-26], molecularly doped polymers[27,28], oligomers [29], and hybrid organic-inorganic perovskite films[30]. Here, we use TSL to characterize the DOS in spin-coated films of two series of organic semiconducting materials of different polarity, based on small-molecule carbazole-biphenyl (CBP) derivatives: $\mathrm{CBP}, \mathrm{mCBP}$, and $\mathrm{mCBP}-\mathrm{CN}$ (series 1) and carbazole-phenyl (CP) derivatives: $\mathrm{mCP}$ and $\mathrm{mCP}-\mathrm{CN}$ (series 2), the chemical structures of which are shown in Figure 1. These materials are widely used as hosts for blue triplet emitters or for emitters based on thermally activated delayed fluorescence (TADF) in organic light-emitting diodes (OLEDs), due to their wide bandgap and high triplet energy. They are also used as charge transporting layers in both OLED and hybrid perovskite based light emitting devices. A suitable polarity of these materials is important for optimizing the performance of TADF-based OLED devices.

In our study, the TSL data has been analyzed using the Gaussian disorder formalism, based on a thermal release of charge carriers from the lower energy part of the intrinsic DOS distribution. We find that for 
materials with similar core chemical structure, the DOS broadens with increasing dipole moment, in essentially the same manner for both series of CBP- and CP-derivatives, in agreement with the dipolar disorder model. Systematic differences in the DOS widths are observed between these two different series of derivatives. This is attributed to different non-polar contributions to the DOS of these materials, which are analyzed using a theoretical approach based on the distributed electrostatic model. Large DOS widths were found in strongly polar hosts and good agreement between experimental and computational results proves the intrinsic character of the large energy disorder in these materials. TSL measurements also show that the Gaussian-shaped DOS of CBP and mCBP are both accompanied by a deep exponential tail.

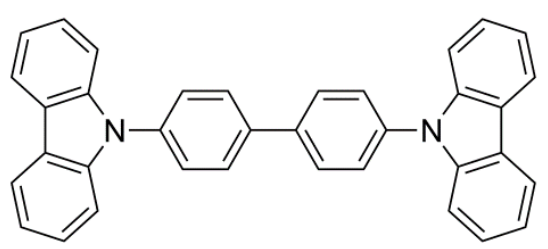

CBP

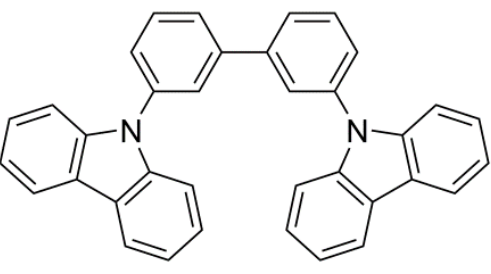

$\mathrm{mCBP}$

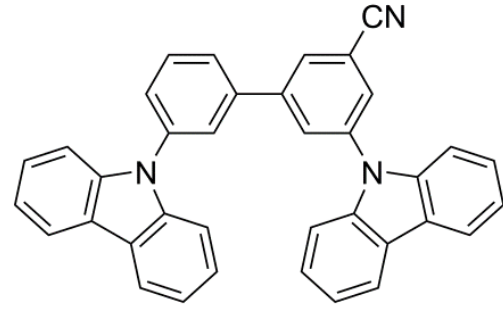

$\mathrm{mCBP}-\mathrm{CN}$<smiles>c1ccc(-n2c3ccccc3c3ccccc32)cc1</smiles>

$\mathrm{mCP}$

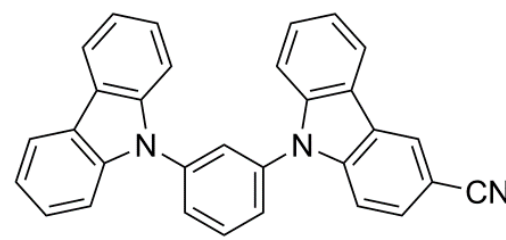

mCP-CN

Figure 1. Molecular structures of the compounds used in this study. CBP-derivatives: 4,4'-Bis( $\mathrm{N}$ carbazolyl)-1,1'-biphenyl (CBP), 3,3'-Di(9H-carbazol-9-yl)-1,1'-biphenyl (mCBP), and 3',5-di(9Hcarbazol-9-yl)- [1,1'-biphenyl]-3-carbonitrile (mCBP-CN). CP-derivatives: 1,3-Bis(N-carbazolyl)benzene (mCP) and 9-(3-(9H-carbazol-9-yl)phenyl)-9H-carbazole-3-carbonitrile (mCP-CN).

\section{Experimental}

\subsection{Materials}

$\mathrm{CBP}, \mathrm{mCBP}, \mathrm{mCBP}-\mathrm{CN}, \mathrm{mCP}$, and $\mathrm{mCP}-\mathrm{CN}$ were purchased from Lumtec Corp. and used as received. Thin films of the above compounds were spin-coated from $20 \mathrm{mg} / \mathrm{ml}$ chloroform solutions onto cleaned quartz substrates (1000 rpm, $30 \mathrm{~s})$ that resulted in typically $150-\mathrm{nm}$-thick layers. Subsequently, the deposited films were dried in oven at $40{ }^{\circ} \mathrm{C}$ for $10 \mathrm{~m}$ and then in a vacuum for $2 \mathrm{~h}$ to remove residual solvent. 


\subsection{TSL techniques}

TSL measurements were carried out over a temperature range from 4.2 to $300 \mathrm{~K}$ with accuracy better than $0.1 \mathrm{~K}$ using an optical temperature-regulating liquid helium bath cryostat designed and fabricated by the Cryogenic Technologies Laboratory (http://www.iop.kiev.ua/ cryo/index en.php) at the Institute of Physics of National Academy of Sciences of Ukraine. In this type of cryostat, the sample is attached to the end of a sample manipulator and is suspended in helium heat-transfer gas environment inside the inner sample chamber. The sample temperature is controlled by the temperature controller unit in a twofold manner: (i) via two separate heating elements (one is integrated into the sample holder, directly heating the sample and the second one is mounted in the sample chamber, heating the heat-transfer gas), and (ii) by regulating the helium flow between the helium bath and the sample chamber via a control of the He gas pressure in the sample chamber.

In TSL experiment, samples were cooled down to $4.2 \mathrm{~K}$ and irradiated for $30 \mathrm{~s}$ with $\lambda_{\mathrm{exc}}=313 \mathrm{~nm}$ light (cw-excitation, light power density was about $5 \mathrm{~mW} / \mathrm{cm}^{2}$ ) selected by appropriate set of cut-off filters from a high-pressure 250W Hg-lamp. No sample heating occurred during UV light illumination and the sample was immersed in liquid helium. The studied organic films did not exhibit any notable photodegradation during the measurements. TSL measurements were done as follows: First, after terminating the photoexcitation, the samples were kept in dark at a constant temperature $T=4.2 \mathrm{~K}$ during a certain dwell time (typically for $10 \mathrm{~min}$ ) in order to allow all isothermal emission processes, like phosphoresce and isothermal recombination of short-range geminate charge-carrier pairs, to decay to negligible level. Then TSL was detected upon heating the sample and TSL emission was detected in a photon-counting mode with a cooled photomultiplier positioned next to the cryostat window.

TSL measurements were performed in two different regimes: uniform heating at the constant heating rate $\beta$ of $0.15 \mathrm{~K} / \mathrm{s}$ and under the fractional heating regime. The details of the TSL measurements have been described elsewhere $[21,22,27]$. The method of fractional TSL avoids the disadvantages of the common glow curve methods based on the constant heating rate - it is characterized by greater accuracy and a high resolving power, and it does not require a knowledge of the frequency factors and re-trapping probabilities [31,32]. The low-temperature fractional TSL was extensively applied by our group to investigate the charge-carrier trapping in a great variety of organic semiconductor materials. The fractional heating TSL technique (also called the fractional glow technique), originally proposed by Gobrecht and Hofmann [32] is an extension of the initial rise method and is based on cycling the sample with a large number of small temperature oscillations superimposed on a uniform heating ramp. The main reason for applying this method is that the usual quantitative evaluation of the TSL glow curves is very inaccurate, or even impossible, if the traps are continuously distributed in energy or if there are several types of traps with discrete but very close lying activation energies. In this case, the glow peaks 
fuse together, and individual glow maxima may not be discernible. The mean activation energy $\langle E\rangle$ is determined during each temperature cycle with a temperature change, $\Delta T$, as

$$
\langle E\rangle(T)=-\frac{d[\operatorname{lnI}(T)]}{d(1 / k T)} ; \text { at } \beta=\text { const, } \Delta T \ll T, n \ll n_{\text {tot }}
$$

where $I(T)$ is the intensity of the TSL, $T$ is the temperature, $k$ is the Boltzmann constant. Here $n$ is number of charge carriers released during each temperature cycle, and $n_{\text {tot }}$ is the total number of carriers trapped by the same sort of traps. These conditions determine the so-called "initial rise method" which is actually the basis for Eq.(1). Since a temperature oscillation, $\Delta T$, is usually much less than the mean value of $T$, the $\langle E\rangle$ could be assumed as equal to $E(T)$, the activation energy of traps emptied at the temperature $T$. A trap distribution function, $H(E)$, can be determined in arbitrary units as [31-33]:

$$
H(E) \propto \frac{I(E)}{d\langle E\rangle / d T}
$$

where $I(E)$ is TSL after converting the temperature scale to the energy scale by means of empirically accessible $\langle E\rangle(T)$ dependence obtained by Eq. (1). As one can see from Eq.(2), in the case when $\langle E\rangle(T)$ is a linear function, the TSL intensity dependence $I(T)$ is an exact replica of populated trap distribution function [19]. The frequency factor at the maximum of TSL peak, $\langle S\rangle$ is given by the formula:

$$
\langle S\rangle=\left\langle E_{m}\right\rangle \beta / k T_{m}^{2} \times \exp \left(\left\langle E_{m}\right\rangle / k T_{m}\right)
$$

where $T_{m}$ and $\left\langle E_{m}\right\rangle$ is the temperature and the activation energy of the maximum of the TSL peak, respectively. All measurements were done in the helium atmosphere.

\subsection{Computer simulations:}

For morphology simulations, we adapt the OPLS-AA force field [34-36]. All Lennard-Jones parameters are taken from this force field and we use the OPLS combination rules and the fudge-factor of 0.5 for 1-4 interactions. Atomic partial charges are computed via the CHELPG scheme [37]. We partition the molecules into rigid fragments: carbazoles and bridge groups in between. These rigid fragments are orientated with respect to one another, such that conformers exhibit different energies, as a result of different electrostatic interactions. The dihedral interaction potentials that connect these fragments are parameterized as described elsewhere [38]: for each fixed value of the dihedral angle the geometry is optimized at the m06-2x/6-311g(d,p) level. The potential difference is then fitted to the RyckaertBelleman polynomial, $V_{r b}(\theta)=\sum_{n=0}^{5}(\cos \theta)^{n}$.

The long-range electrostatic interactions are treated by using a smooth particle mesh Ewald technique. A cutoff of $1.3 \mathrm{~nm}$ is used for the non-bonded interactions. The equations of motion are integrated with 
a time-step of 0.002 ps. All molecular dynamics simulations (MD) are performed in the NPT ensemble using the canonical velocity-rescaling thermostat [39] and the Berendsen barostat [40] as implemented in the GROMACS simulation package [41,42].

To obtain the amorphous morphology, 3000 molecules are pre-arranged on a lattice and compressed (anisotropic NPT barostat) at $T=800 \mathrm{~K}$ for $1 \mathrm{~ns}$. The system is then cooled to $300 \mathrm{~K}$ during a $1 \mathrm{~ns}$ run. Fast cooling freezes the isotropic orientation of the high-temperature liquid, leading to an amorphous molecular ordering.

Using the molecular dynamics trajectories, we evaluate the anion, cation, and neutral state energies for each molecule in a morphology using a perturbative approach [43-45]. In this approach, the total energy is a sum of the gas-phase, electrostatic, and induction contributions, $E_{e, h, n}=E_{e, h, n}^{\mathrm{gas}}+E_{e, h, n}^{\mathrm{stat}}+E_{e, h, n}^{\mathrm{ind}}$. To evaluate the electrostatic contribution, we used distributed atomic multipoles up to the fourth order. The induction contributions to site energies is calculated self-consistently using the Thole mode $[46,47]$ on the basis of the atomic polarizabilities and distributed multipoles obtained by using GDMA program 48] for a cation, anion, and a neutral molecule. All calculations were performed using the aperiodic Ewald summation scheme [45] as implemented in the in-house developed VOTCA package [49].

\section{Results}

\subsection{DOS probing by TSL measurements}

Surprisingly strong thermoluminescence signals induced by UV radiation at liquid helium temperature were observed in neat thin films made of both CBP- and CP- derivatives studied in the present work. We first consider TSL measurements in thin films of CBP-derivatives. A typical TSL glow curve of a mCBP-CN film measured after photoexcitation with $313 \mathrm{~nm}$ at $4.2 \mathrm{~K}$ is presented in Figure $2 \mathrm{a}$ and it reveals a broad, slightly asymmetric band, with the maximum at $T_{m} \cong 93 \mathrm{~K}$. The asymmetric shape of the TSL band might be due to overlapping of the main high-temperature peak at 93K, with a weaker lowtemperature peak at around $40 \mathrm{~K}$, as depicted by curves $1^{\prime}$ and $1 "$ in Figure 2a. The TSL signal is observed immediately as the sample is heated and extends to $\sim 220 \mathrm{~K}$, as expected for a disordered material devoid of deep charge-carrier traps. It is qualitatively similar to TSL phenomena observed in other organic disordered systems studied before [18-23], and can be ascribed to detrapping of charge carriers from the localized states that occupy the lower energy part of the intrinsic DOS distribution, schematically shown in the inset to Figure 2a. The fact that TSL in $\mathrm{mCBP}-\mathrm{CN}$ films extends to moderately high temperatures, is evident of relatively strong energy disorder in this material, compared, for instance, to conventional semiconducting polymers, such as a methyl-substituted ladder-type poly(para- 
phenylene) (MeLPPP) [18] and a poly-fluorene (PF) derivative [20] with reduced energy disorder. Yet, it is closer to the TSL peak observed before in TSL of vacuum deposited films of $\mathrm{AlQ}_{3}$.
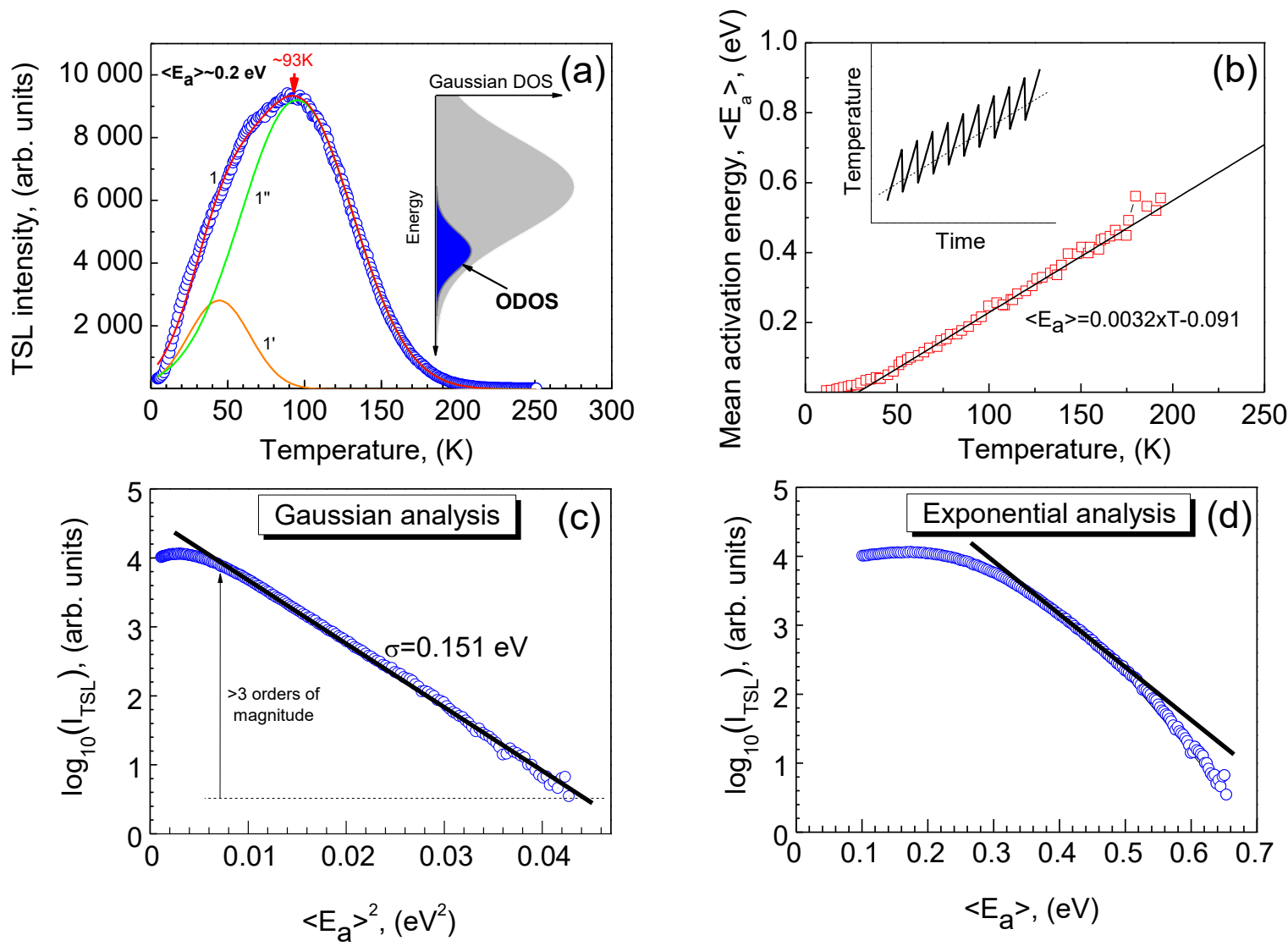

Figure 2. (a) Spectrally integrated TSL glow curve measured after excitation with $313 \mathrm{~nm}$-light for 3 min at $4.2 \mathrm{~K}$ in mCBP-CN film. Curves 1 ' and 1" represent deconvolution of the TSL band (curve 1) into two Gaussians. Inset shows schematic DOS distribution together with the distribution of trapped charge-carriers at liquid-helium temperature after their energy relaxation. Temperature dependence of the mean activation energy $\left\langle\boldsymbol{E}_{\boldsymbol{a}}\right\rangle$ as obtained by fractional TSL (symbols) and extrapolation by an empirical expression (4) (solid line). Inset shows a schematic picture to illustrate principle of fractional TSL regime when temperature oscillations are superimposed on a uniform heating. (c) Gaussian analysis of the high-energy wing of the TSL curve (see text for details). (d) Same data as in (c) but plotted in exponential representation.

Similar to previously studied photoconducting polymers [18-22,27], the mCBP-CN film is characterized by a quasicontinuous trap distribution, with a mean activation energy $\left\langle E_{a}\right\rangle$ that is linearly increasing with temperature (Figure $2 \mathrm{~b}$ ), as revealed by fractional TSL measurements (see the Methods Section for details). The temperature dependence of $\left\langle E_{a}\right\rangle$ (in eV) can be described by the following empirical relation: 


$$
\left\langle E_{a}\right\rangle(T)=0.0032 \times T-0.091 .
$$

The empirical expression given by Eq. (4) supports the basic formula of Simmons-Taylor theory [51] for thermally-stimulated current (TSC), which predicts a qualitatively similar linear temperature dependence of the apparent activation energy. It is worth noting that such kind of linear relation for $\left\langle E_{a}\right\rangle(T)$ has also been justified by analytical variable range-hopping calculations for disordered organic semiconductors $[18,19]$. The mean activation energy and frequency-to-escape factor at the maxima of the TSL peak $T_{m} \sim 93 \mathrm{~K}$ are: $\left\langle E_{a}\right\rangle \cong 0.2 \mathrm{eV}$ and $\langle S\rangle=2.7 \times 10^{9} \mathrm{~s}^{-1}$, respectively. Meaning of the frequency-toescape factor in disordered solids will be discussed in more details in the Discussion section.

Using an empirical calibration Eq.(4) to convert the temperature scale to a trap energy scale, and then plotting the high-temperature part of the TSL signal intensity logarithmically either against $\left\langle E_{a}\right\rangle^{2}$ or $\left\langle E_{a}\right\rangle$ allows for a "Gaussian-" or an "Exponential analysis", respectively, as shown in Figure 2c and 2d. It proves that the high-temperature wing of a TSL curve becomes a straight line when plotting $\ln \left(I_{T S L}\right)$ vs. $\left\langle E_{a}\right\rangle^{2}$ (Figure 2c). It is remarkable to see an almost perfect Gaussian fit of the high-temperature wing of the measured curve observed over three decades of TSL intensity variation (Figure 2c), whereas the same data plotted in the $\log \left(I_{T S L}\right)$ vs. $\left\langle E_{a}\right\rangle$ representation shows clear deviation from an exponential dependence (Figure 2d). This observation provides strong support for a Gaussian shape of the DOS profile in this material. The slope of the straight line in Figure $2 c$ is a measure of the DOS width. The energy disorder parameter $\sigma=0.151 \mathrm{eV}$ was obtained for the $\mathrm{mCBP}-\mathrm{CN}$ film, which is very close to the disorder parameter $\sigma=0.15 \mathrm{eV}$ estimated for $\mathrm{AlQ}_{3}$ films [52] by charge transport measurements. Such a relatively strong energy disorder is well expected for polar materials - the dipole moment for $\mathrm{AlQ}_{3}[52]$ and $\mathrm{mCBP}-\mathrm{CN}$ is 4.9 and 4.6 D, respectively (Table 1). It is worth noting that the above Gaussian analysis used here is conceptually similar to that earlier employed by Bässler [53] and Eiermann et al. [54] using the TSC technique for studying the width of the DOS distribution in disordered tetracene layers, by analyzing the shape of the high-energy wing of a TSC peak.

Figure 3a compares normalized TSL curves measured in CBP, mCBP, and $\mathrm{mCBP}-\mathrm{CN}$ films under the same conditions. All of these host materials revealed a characteristic TSL peak, which featured a clear shift and progressive broadening towards higher temperatures with increasing molecular dipole moment (Figure 3a). The TSL peak maximum (depicted by arrows in Figure 3a) also shifts from $T_{m} \cong 72 \mathrm{~K}$ for CBP to $T_{m} \cong 78$ and $93 \mathrm{~K}$ for $\mathrm{mCBP}$ and $\mathrm{mCBP}-\mathrm{CN}$ films, respectively (Table 1 ). The latter effect is a clear sign of increasing energy disorder in this sequence of host materials from the same series of CBP-derivatives. The shift is a consequence of the fact that the mean activation energy, which carriers reach in the course of their downward hopping relaxation at very low temperature within a Gaussian DOS, is directly proportional to energy disorder parameter $\sigma$ [55]. 

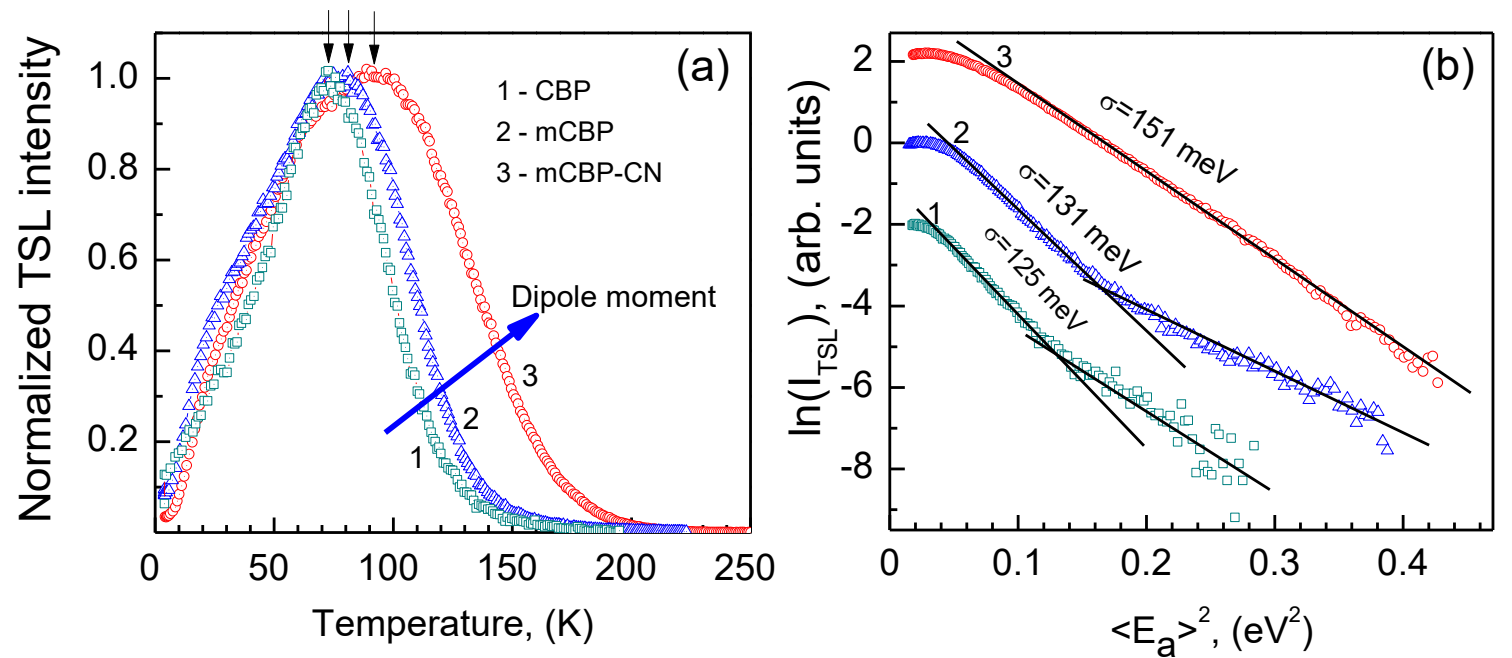

Figure 3. a) Normalized TSL glow curves measured after excitation with $313 \mathrm{~nm}$-light for $3 \mathrm{~min}$ at 4.2 $\mathrm{K}$ in $\mathrm{CBP}, \mathrm{mCBP}$ and $\mathrm{mCBP}-\mathrm{CN}$ films (curve 1, 2, 3, respectively); b) Gaussian analysis of high temperature wings of TSL curves shown in (a) (see text for details). Curves are vertically shifted with respect to each other for clarity.

A Gaussian analysis of the high temperature wings of TSL curves for CBP, mCBP, and mCBP-CN films, using Eq.(4) as described above, is shown in Figure 3b. The plot reveals a Gaussian dependence in all samples - its slope yields the width of the DOS, which increases with increasing dipole moment from $0.125 \mathrm{eV}$ for virtually non-polar CBP ( $p \cong 0 \mathrm{D})$, to 0.131 and $0.151 \mathrm{eV}$ for $\mathrm{mCBP}(p=2.15 \mathrm{D})$ and $\mathrm{mCBP}-\mathrm{CN}$ $(p=4.6 \mathrm{D})$, respectively (cf. Table 1 ). This points to an important role of the dipolar disorder effects to the increase of the energy disorder in this series of CBP-derivatives, with increasing dipole moments of constituting molecules. The disorder parameter $\sigma=0.112 \mathrm{eV}[56]$ has been inferred for evaporated CBP films from charge transport measurements, which is - comparable to the $\sigma=0.125 \mathrm{eV}$ we obtained here for the spin-coated CBP film.

A remarkable observation of this study is that $\mathrm{CBP}$ and $\mathrm{mCBP}$ films also revealed a slower decaying deep exponential tail at the lowest portion of the DOS, following the Gaussian distribution. Fitting the deep tail with exponential distribution $g(\epsilon) \propto \exp \left(\epsilon / k T_{0}\right)$, where $T_{0}$ is the characteristic width of the exponential distribution (for energies $\epsilon<0$ ), yields 716 and $673 \mathrm{~K}$, for CBP and mCBP films, respectively (Table 1). 
Table 1 . Dipole moment $(p)$, polarizability of neutral molecule $\left(\alpha_{\text {neutr. }}\right)$, polarizability of cation ( $\left.\alpha_{\text {cation. }}\right)$, width of Gaussian DOS $(\sigma)$ evaluated by TSL and by computer simulations; dipolar disorder component $\left(\sigma_{\text {dip }}\right)$ estimated from TSL data, and width of superimposed exponential low-energy tail $\left(T_{0}\right)$ evaluated from TSL data.

\begin{tabular}{|c|c|c|c|c|c|c|c|}
\hline Material & $\begin{array}{c}\text { Dipole } \\
\text { moment } \\
p(\mathrm{D})\end{array}$ & $\begin{array}{c}\alpha_{\text {neutr. }} \\
\left(\mathrm{Bohr}^{3}\right)\end{array}$ & $\begin{array}{c}\alpha_{\text {cation }} \\
\left(\mathrm{Bohr}^{3}\right)\end{array}$ & $\begin{array}{c}\text { DOS width } \\
(\mathrm{TSL}) \\
\sigma(\mathrm{eV})\end{array}$ & $\begin{array}{c}\sigma_{\text {dip }} \\
(\mathrm{TSL}) \\
(\mathrm{eV})\end{array}$ & $\begin{array}{c}T_{0}(\mathrm{~K}) \\
(\mathrm{TSL}) \\
(\mathrm{K})\end{array}$ & $\begin{array}{c}\text { DOS width } \\
(\text { simulations }) \\
\sigma(\mathrm{eV})\end{array}$ \\
\hline $\mathrm{CBP}$ & $0.04^{*}$ & $670^{\ddagger}$ & $3200^{\ddagger}$ & 0.125 & 0 & $716 \mathrm{~K}$ & 0.10 \\
\hline $\mathrm{mCBP}$ & $2.15^{*}$ & $600^{\ddagger}$ & $3000^{\ddagger}$ & 0.131 & 0.039 & $673 \mathrm{~K}$ & 0.12 \\
\hline $\mathrm{mCBP}-\mathrm{CN}$ & $4.6^{*}$ & $600^{\ddagger}$ & $3000^{\ddagger}$ & 0.151 & 0.085 & - & 0.20 \\
\hline $\mathrm{mCP}$ & $1.35^{*}$ & $450^{\ddagger}$ & $1500^{\ddagger}$ & 0.140 & 0.024 & - & 0.16 \\
\hline $\mathrm{mCP}-\mathrm{CN}$ & $6^{*}$ & $540^{\ddagger}$ & $1500^{\ddagger}$ & 0.177 & 0.111 & - & 0.24 \\
\hline
\end{tabular}

* calculated by using M062X functional in density functional theory;

‡ calculated using a perturbative approach as implemented in the Gaussian 09 package (opt=polar) keyword (https://gaussian.com/polar/).

Next, we compare the TSL properties of the above CBP-derivatives with another series of host materials, based on CP-derivatives. TSL glow curves measured under the same conditions in $\mathrm{mCP}$ and $\mathrm{mCP}-\mathrm{CN}$ films are shown in Figure 4a (curve 1 and 2, respectively). Although these films exhibit a TSL signal within a similar temperature range from 4.2 to $\sim 200 \mathrm{~K}$ as the CBP-derivatives (cf. Figure 3a), the shape of their TSL curves are amazingly different. The TSL curve of mCP clearly consists of two overlapping peaks with $T_{m}$ at 25 and $65 \mathrm{~K}$, respectively, as shown by deconvolution of the measured TSL curve into two Gaussian peaks (curves 1' and 1" in Figure 4). It is noteworthy that the low-temperature feature with $T_{m}$ $=25 \mathrm{~K}$ dominates in the intensity. A similarly shaped TSL is found in the strongly polar $\mathrm{mCP}-\mathrm{CN}$ film ( $p=6$ D) (Figure 4a, curve 2) - it also consists of two overlapping peaks, yet shifted towards higher temperatures with $T_{m}$ at 33 and $78 \mathrm{~K}$. We should, however, note that a low-temperature feature is also noticeable in TSL curves of $\mathrm{mCBP}$ and $\mathrm{mCBP}-\mathrm{CN}$ films, but it is significantly weaker and seen as just a weak shoulder (cf. curve 1' in Figure 2a). Our complementary studies have demonstrated that the lowtemperature TSL feature in these materials arises due to frustrated energy relaxation [57] of chargecarriers photogenerated at $5 \mathrm{~K}$, and therefore, some of them get stuck in the upper portion of the DOS; but this effect will be explored in greater details in future works of the authors. Since just the deeper 
portion of the DOS profile controls the charge transport in amorphous organic semiconductors, the high-temperature TSL feature peaking at 65 and $78 \mathrm{~K}$ in $\mathrm{mCP}$ and $\mathrm{mCP}-\mathrm{CN}$, respectively, is expected to be of practical relevance (we refer to it as the 'main TSL peak') and thus is analyzed below.
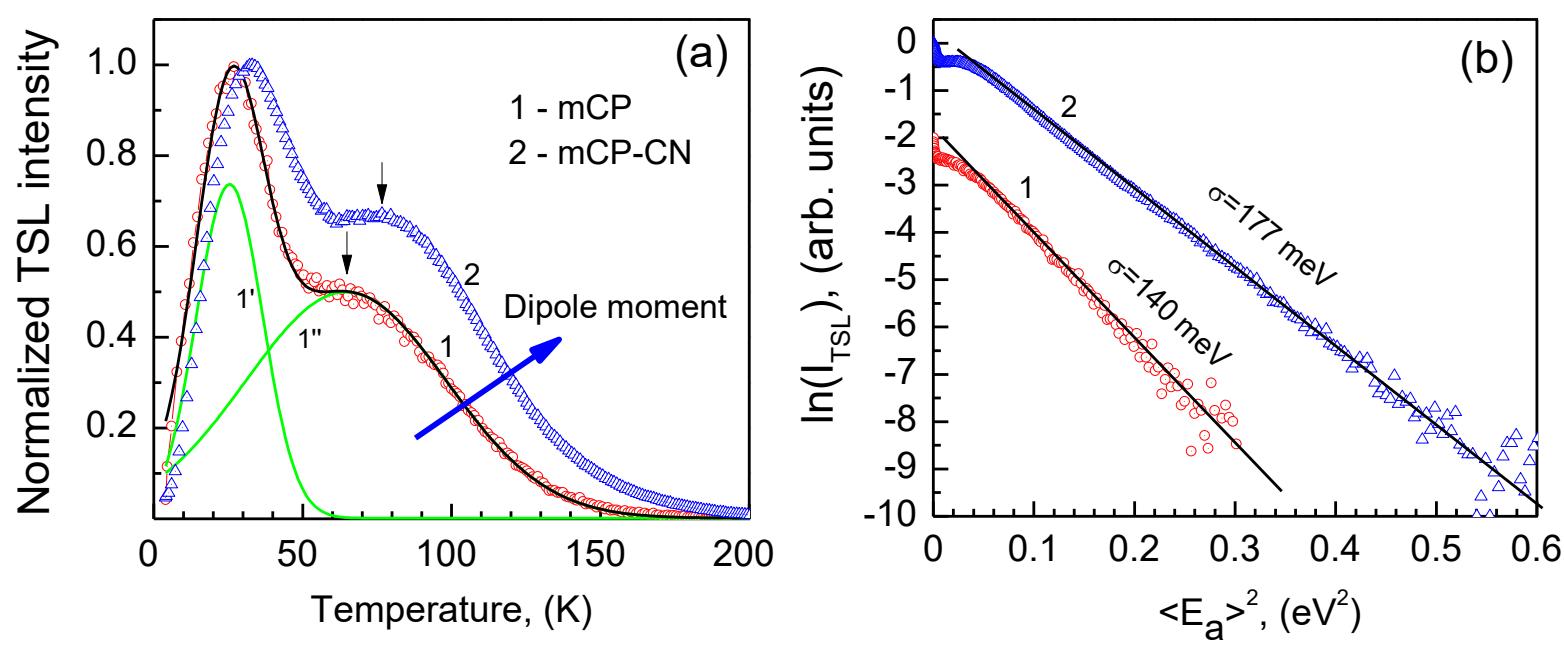

Figure 4. a) Normalized TSL glow curve measured after excitation with 313nm-light for 3 min at 4.2 $\mathrm{K}$ in $\mathrm{mCB}$ and $\mathrm{mCP}-\mathrm{CN}$ film (curve 1 and 2, respectively). Curves 1 ' and 1" represent deconvolution of the TSL curve of mCP (curve 1) into two Gaussian peaks; b) Gaussian analysis of high temperature wing of the corresponding TSL curves shown in (a) (see text for details).

The mean activation energy $\left\langle E_{a}\right\rangle$ was measured in $\mathrm{mCP}$ and $\mathrm{mCP}-\mathrm{CN}$ films as a function of temperature by fractional TSL and in both materials it exhibited a similar temperature dependence as that given by Eq.(4). The Gaussian analysis of the high temperature wings of TSL curves for $\mathrm{mCP}$ and $\mathrm{mCP}-\mathrm{CN}$ films was done as described above for other host materials and is presented in Figure $4 \mathrm{~b}$ by curve 1 and 2, respectively. The energy disorder parameter $\sigma$ in these materials was inferred from the slope of the high-temperature wing of TSL curves plotted in a Gaussian representation: $\ln \left(I_{T S L}\right)$ vs. $\left\langle E_{a}\right\rangle^{2}$. Both materials show a nice Gaussian fit of the high-temperature wings of the measured curves over several decades of TSL intensity variation. Since the TSL signal in mCP-CN film was significantly stronger than in $\mathrm{mCP}$, we were able to extend its Gaussian analysis towards deeper energies (4b, curve 2). The results in Figure $4 b$ demonstrates a clear impact of the magnitude of dipole moments on the width of the DOS, which increases from $\sigma=0.140 \mathrm{eV}$ for moderately polar mCB ( $p=1.35 \mathrm{D}$ ) to $0.177 \mathrm{eV}$ in strongly polar $\mathrm{mCP}-\mathrm{CN}(p=6 \mathrm{D})$. This suggests a rather significant energy disorder inherent in CBderivatives. 


\subsection{Computation of the DOS distribution}

To provide deeper insights into the DOS structure and independent verification of the DOS parameters probed by the TSL technique in the above OLED host materials, we carried out computer simulations. To evaluate the DOS distribution of the amorphous systems, we first employed atomistic molecular dynamics (MD) simulations to generate the amorphous morphologies, as described in the Methods Section. We then used quantum chemical calculations and polarizable force fields to compute site energies. Using the simulated amorphous morphologies, site energies of all molecules were evaluated by using the parameterized (see the Methods Section) polarizable force fields. The corresponding DOS, shown in Figure 5, have Gaussian shapes with variances $\sigma$.

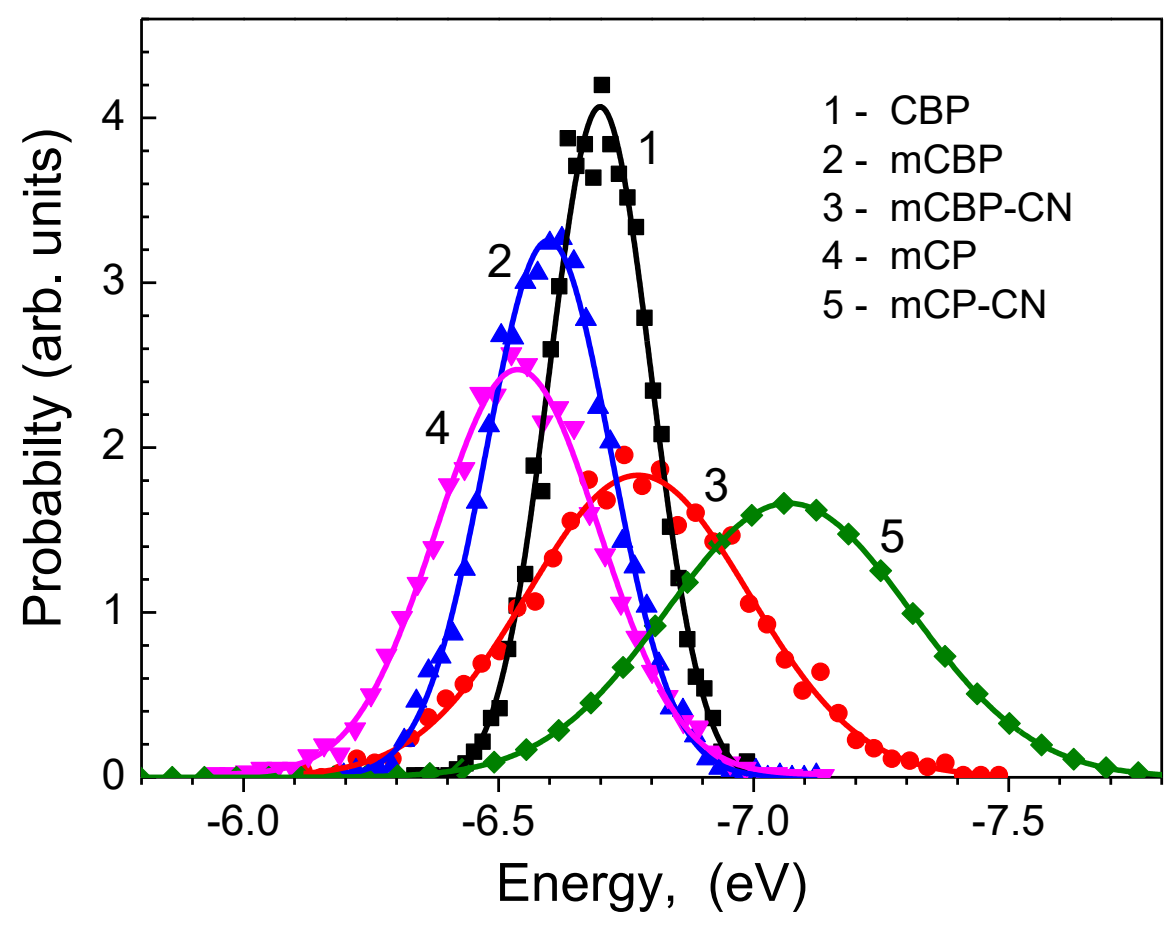

Figure 5. DOS distributions calculated for $C B P, m C B P, m C B P-C N$, and $m C P$ (curves 1, 2, 3, and 4, respectively) employing molecular dynamics simulations. Solid lines depict a Gaussian fit to the data.

The DOS widths $\sigma$ inferred from the simulation results are listed in Table 1. Despite the similarity in chemical structures of CBP-derivatives, the energy disorder varies over a broad range in these materials from $0.10 \mathrm{eV}$ to 0.12 and $0.20 \mathrm{eV}$ for $\mathrm{CBP}, \mathrm{mCBP}$, and $\mathrm{mCBP}-\mathrm{CN}$, respectively. As expected, the smallest energy disorder is found in CBP films and this can be readily explained by the symmetric molecular structure: its ground state dipole moment is virtually zero. As a result, the first non-vanishing electrostatic contribution is due to the interaction of the charge carrier and the quadrupole moments of the surrounding molecules. Since this contribution is much smaller than the charge-dipole interaction, the energetic disorder of these compounds is relatively small, of the order of $0.1 \mathrm{eV}$. The shift of the 
ionization potential in a solid state is mostly due to the induction stabilization, and the shape of the density of states is Gaussian. The dipole moment increases sequentially in mCBP and mCBP-CN molecules, which gives rise to the increase of charge-dipole integration and hence to larger electrostatic contribution to the energetic disorder in these materials. This verifies that the dipolar disorder is responsible for the significant increase of the energy disorder within the series of CBP-derivatives. Such conclusion is, however, valid for compounds of the same family, in our case, with similar molecular polarizabilities. Indeed, the DOS simulations confirmed that the CBP, $\mathrm{mCBP}, \mathrm{mCBP}-\mathrm{CN}$ family has similar polarizability of cations and neutral molecules (see Table 1), hence their DOS width increases with the molecular dipole moment. $\mathrm{mCP}$ has a significantly smaller polarizability of the cation compared to mCBP ( 1500 vs. $\sim 3000 \mathrm{Bohr}^{3}$, see Table 1). Therefore, the screening of the electrostatic disorder by the induction interactions is smaller than that for $\mathrm{mCBP}$, giving rise to a DOS width of $0.16 \mathrm{eV}$ for $\mathrm{mCP}$.

It is pertinent to note that dipole moments can vary considerably with molecular conformations. Different conformers lead to variations in the molecular dipole moment in the amorphous morphologies. The distribution of dipole moments in the morphology inferred from MD simulations normally features a fairly broad peak [75] around a certain value that can, to a certain extent, be used as the 'average' dipole moment. We are not claiming that this trend is universal for all organic semiconductors, especially taking into account that the distributions of dihedral angles are sensitive to the deposition protocols. However, in our host materials, the average dipole moments inferred from MD simulations [75] were found to correlate well with those shown in Table 1 and used for the analysis depicted in Fig. 6.

\section{Discussion}

The DOS widths determined by the TSL techniques are in reasonable agreement with the computation results, (cf. Table 1) predicting a significant variation of the energy disorder among the considered host materials. Although the obtained experimental values somewhat deviate from the simulated $\sigma$ values mostly in systems with strong energy disorder, they do demonstrate a remarkably similar trend in the variation of energetic disorder among these materials. The reason for discrepancy between the widths of the DOS probed by TSL and that obtained by computer simulations should be a subject of further research.

Comparison of the DOS parameters determined by TSL and by computer simulations (Fig. 5) has two important implications: (i) It confirms that the large energetic disorder observed experimentally (ranging from $\sigma=0.125$ to $0.177 \mathrm{eV}$ ) characterizes the intrinsic DOS, i.e., the DOS of a chemically pure disordered material, rather than that affected by impurity-related traps. Therefore, the charge transport in such 
OLED hosts is expected to be inherently very dispersive. (ii) It reveals the utmost importance the polarizability of the cation to the DOS width, which is a key finding in the present study. As attested by the computer simulations, the physical reason for the enlarged energetic disorder in CP-derivatives compared to CBP ones can be attributed to much smaller polarizability of their cation states, owing to a more rigid chemical structure of these molecules, and thus smaller screening effect.

The calculated DOS distributions in Fig. 5 yield not only the variances $\sigma$ of their Gaussian shapes, but also energy position of HOMO levels. On the other hand, methods based on thermally activated spectroscopy, like TSL or TSC, use thermal energy generated by heat for probing localized states occupied with charge-carriers within intrinsic (or extrinsic) DOS. Since such energy is much less than energy corresponding to HOMO-LUMO gap, they can only characterize the DOS parameters. Nonetheless, in host-guest compositions, TSL can potentially yield the energy difference between corresponding HOMO or LUMO levels of host and guest materials, provided that guest molecules create in the host material a hole or an electron trap, respectively.

\subsection{Effect of dipole moments}

Next, we discuss the effect of dipole moments, of the materials studied here, on the width of the DOS evaluated by the TSL technique. As it has long been suggested, the total width $\sigma$ in a polar amorphous organic solid can be decomposed into a dipolar component $\sigma_{\text {dip }}$ and a so-called van der Waals component $\sigma_{v d W}[3,58]$. If different energy disorder contributions are independent and follow Gaussian statistics, then the total width is

$$
\sigma^{2}=\sigma_{w d W}^{2}+\sigma_{d i p}^{2}
$$

The term $\sigma_{v d W}$ in Eq.(5) is used here to describe the whole non-dipolar disorder component, despite its physical origin being solely attributed to the variation of the interaction of a charged molecule with induced dipole moments in the molecular environment $[59,60]$. Borsenberger and Weiss [3] proposed that fluctuations of relative orientations and intermolecular distances between the hopping site and surrounding molecules, create fluctuation of the induced dipolar cloud in the nearby molecules, which makes the major contribution to $\sigma_{v d W}$. Subsequently, it was later recognized that quadrupole moments can be sufficiently high in non-polar materials with zero dipole moment and hence variation of chargequadrupole interactions can also contribute to the energy disorder $[9,61]$. Therefore, to adhere to established terminology, we imply here that $\sigma_{v d W}$ encompasses contributions from all sources of the energy disorder, excluding the dipolar contribution. In other words, it describes the total energy disorder in a non-polar system. 
The dipolar disorder $\sigma_{\text {dip }}$ contributes strongly to the overall energy disorder in polar organic solids and it leads to a stronger DOS broadening compared to the charge-quadrupole interaction [61]. $\sigma_{d i p}$ arises due to randomly oriented permanent molecular dipoles that generate fluctuation in the electrostatic potential due to charge-dipole interactions, in addition to the disorder that is already present in nonpolar systems which leads to DOS broadening [3]. Dieckmann et al. [58] first obtained an expression for the dipolar component using Monte-Carlo numerical calculations. Subsequent analytic calculation by Young [62] has confirmed the Gaussian form of the DOS in polar systems and the relation for $\sigma_{\text {dip }}$ was derived as

$$
\sigma_{\text {dip }}=\frac{7.04}{a^{2} \varepsilon} c^{1 / 2} p \quad(\text { in eV })
$$

where $a$ is the intersite distance (lattice constant) in Angstrom, $\varepsilon$ the dielectric constant, $c$ the fraction of the lattice occupied by dipoles (in our case $c=1$ ), and $p$ is the dipole moment in Debye. Although, as it was recently recognized [63] that accounting for the individual molecular polarizabilities and nearest neighbor interactions might lead to deviation from the suggested $1 / \varepsilon$ dependence in Eq. (6), the dipolar disorder $\sigma_{\text {dip }}$ is predicted to scale linearly with dipole moment. An analysis based on Eq.(6), in conjunction with (1), has been widely used to evaluate the dipolar disorder contribution to the overall energy disorder from the charge transport measurements in small-molecule organic glasses $[3,64-66]$ and molecularly doped polymers, [3,60,67-69] with different dipole moments and dipole concentration, that justifies the applicability of Eq.(6).

Assuming the $\sigma_{v d W}$ component is a constant for materials within the same series of derivatives, Eqs. 2 and 3 provides a means for analyzing the effect of dipole moment of different compounds on the total width, by plotting $\sigma^{2}$ versus $p^{2}$. Figure 6 (curve 1 ) clearly shows the predicted linear relationship for $C P B$ - derivatives: $\mathrm{CBP}, \mathrm{mCBP}$, and $\mathrm{mCBP}-\mathrm{CN}$ (solid circles) indicating the justification for the analysis. The non-polar component $\sigma_{v d W}$ for this series of derivatives is equal to the DOS width $\sigma=0.125 \mathrm{eV}$ obtained for non-polar CBP film. Using $\sigma_{v d W}=0.125 \mathrm{eV}$, Eq.(5) yields the dipolar component $\sigma_{d i p}=0.039$ and $0.085 \mathrm{meV}$, for polar mCBP and mCBP-CN, respectively (Table 1). The intersite distance $a=11 \AA$ may be estimated from the slop of the solid line 1 in Figure6 using Eq.(6). It agrees well with the effective lattice constant $a=12$ Å estimated recently for CBP films from charge transport measurements [70]. 


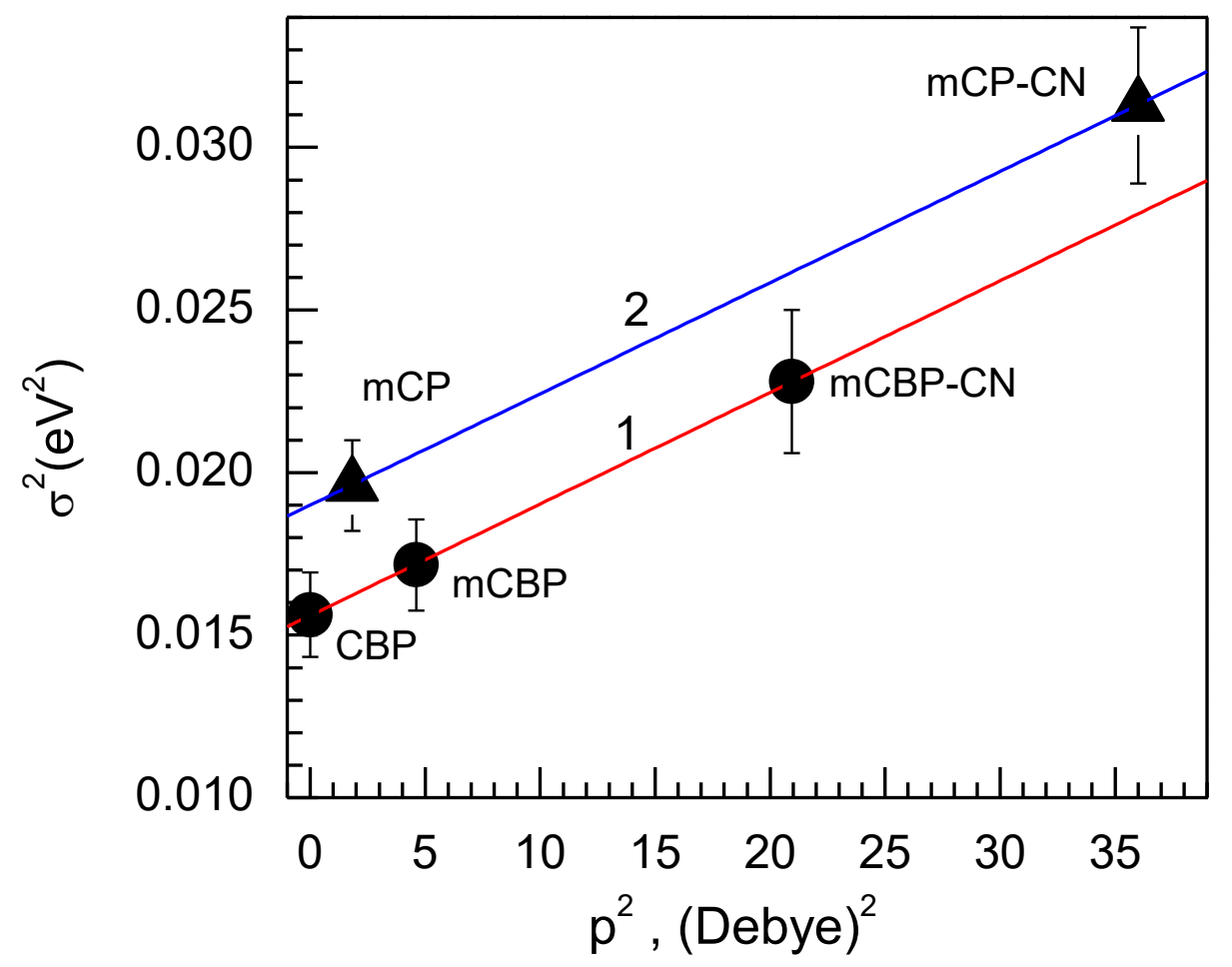

Figure 6. Estimated disorder parameter vs. dipole moment for two series of OLED host materials: CBP- and CP- derivatives (solid circle and triangle symbols, respectively). Solid lines are fitting of the experimental data with Eq.(5) and (6) using $\boldsymbol{c}=1$ and $\boldsymbol{\varepsilon}=3.13$ [71].

A qualitatively similar trend with increasing dipole moment was found for a series of $\mathrm{CP}$-derivatives (Figure 6, curve 2). Although only two host materials (mCP and $\mathrm{mCP}-\mathrm{CN}$ ) of this series were available to us. The energy disorder parameter has demonstrated a strong increase with increasing dipole moment from 1.35 to $6 \mathrm{D}$ for $\mathrm{mCP}$ and $\mathrm{mCP}-\mathrm{CN}$, respectively (Figure 6 , solid triangles). It is remarkable that this dependence in $\sigma^{2}$ vs. $p^{2}$ representation features almost the same slop (curves 2 in Figure 6 ) as the linear dependence obtained for the above CBP-derivatives (curve 1), yet is offset with respect to the latter. These observations suggest that the dipolar disorder component increases in a similar manner with increasing dipole moments for both series of materials, while the non-polar $\sigma_{v d W}$ disorder component differs significantly for them. The $\sigma_{v d W}=0.138 \mathrm{eV}$ was determined for mCP and mCP-CN from the $p^{2}=0$ intercept of curve 2 (Figure 6), which turns out to be significantly large than $\sigma_{v d W}=0.125$ $\mathrm{eV}$ determined for the above series of CBP-derivatives. Using the above $\sigma_{v d W}$ value, Eq.(5) yields $\sigma_{d i p}=$ 0.024 , and $0.111 \mathrm{meV}$, for $\mathrm{mCB}$ and $\mathrm{mCB}-\mathrm{CN}$ films, respectively (Table 1 ). The above findings suggest that the enlarged energy disorder in CP- compared to CBP- derivatives, mostly relates to the intrinsically larger non-dipolar component of the energy disorder.

The correlation of the observed dipole effect on DOS width, determined by TSL technique, with the prediction of the dipolar disorder model [62], is striking. Implying that the OLED host materials considered here, behave similar to conventional amorphous organic photoconductors, previously 
studied by the ToF technique in the form of neat molecular glasses $[3,64-66]$ or as charge transporting molecules in molecularly doped polymers $[3,60,67-69]$. It should be noted that the dipolar disorder model given by Eq.(6) does not consider the variations in the molecular dipole moment caused by different conformers in the amorphous morphologies. However, despite the simplification, we believe this model can provide a good first-order approximation for description of the dipolar-induced DOS broadening in our OLED hosts. Notice that that the dependence presented in Figure 6 and concomitant analysis is generally not a new result for the class of organic semiconducting materials. There are numerous similar findings in literature for the width of the DOS inferred from charge mobility measurements in different polar systems. The new result of the present work is that the same effects were observed using a purely optical experimental approach.

Finally, it worth mentioning that Hoffmann at al. [76] have recently demonstrated that, in contrast to small-molecule organic materials, the inhomogeneous broadening of the DOS for holes and neutral excitons can be remarkably similar in the case of conjugated polymers. This was suggested to be a characteristic feature of conjugated polymers in which disorder results predominantly from the statistical variation of the lengths of the conjugated segments rather than from van der Waals coupling among the chains and can be used for prediction of the DOS for holes from simple optical spectroscopy. This effect was observed for a series of conjugated alternating phenanthrene indenofluorene copolymers [76] and probably more research is needed to confirm such a correlation by involving more copolymer materials.

\subsection{DOS shapes}

Another remarkable finding of this study is (i) direct observation using an optical method (TSL) of a pronounced deep exponential tail that follows a Gaussian distribution, and (ii) that this effect turns out to be material dependent - it is seen in CBP and mCBP films, while mCBP-CN, mCP and mCP-CN host materials feature solely Gaussian profile with a negligible, if any, exponential tail (cf. Figure 3b and 4b). The origin of such qualitatively different DOS distributions in organic solids deserves more thorough investigation, involving more amorphous organic semiconducting materials, going beyond the scope of the present study. However, a deep exponential tail in disordered organic semiconductors is not a surprising result - it has previously been reported using different methods. In a series of publications by the Eindhoven group $[8,72,73]$ they claimed that their drift-diffusion device model provides a much better description of experimental temperature dependent IV-characteristics measured in some AOS materials, if one assumes a combination of a Gaussian density of transport states plus superimposed exponential density of trap states. They assumed that the charge traps can be responsible for the 
exponential tail and suggested the following cumulative DOS in realistic organic semiconducting materials $[8,72,73]$

$$
g(\epsilon)=\frac{N_{t}}{\sqrt{2 \pi \sigma^{2}}} \exp \left(-\frac{\epsilon^{2}}{2 \sigma^{2}}\right)+\frac{N_{t r a p}}{k T_{0}} \exp \left(\frac{\epsilon}{k T_{0}}\right)
$$

where $\sigma$ and $N_{t}$ is the Gaussian DOS width and the density of transport sites, respectively. $T_{0}$ and $N_{\text {trap }}$ are the characteristic width of the exponential distribution (for energies $\epsilon<0$ ) and the density of trap sites, respectively. This idea was supported by Hulea et al. [17] who scanned the DOS distribution of a poly(p-phenylene vinylene) (PPV) film over a wide energy range using an electrochemically gated transistor and found an almost exponential trap distribution at the deep tail superimposed onto an intrinsic Gaussian DOS distribution. Moreover, several KPFM studies [10,11] provided direct evidence that AOS materials exhibit both a central Gaussian DOS and an exponential tail.

Several theoretical explanations have been suggested for the complex DOS distribution observed in AOS. May et al. [9] demonstrated that in a material with molecular dipole moments, exponential tails can develop if its negatively or positively charged sites are strongly polarizable. This results in large induced dipoles on these charged sites and thereby increases their interaction with the polar environment. Such non-linear effects of dipolar fields give rise to strong potential fluctuation and thereby lead to the appearance of an exponential tail. Additionally, the same group has recently found that a HOMO level position below $6 \mathrm{eV}$ and LUMO above $3.6 \mathrm{eV}$, in organic semiconductors can facilitate hole and electron trapping, respectively [74] .

Interestingly, our observations seem to be consistent with the above prediction for a trap-free energy window - virtually no exponential tail was observed in mCP films (cf. Figure 4b), where the HOMO level is at $5.9 \mathrm{eV}$, i.e. within a "trap-free energy window", inside which organic semiconductors normally do not experience charge trapping [74]. The above concept implies that materials with an ionization energy lower than $6 \mathrm{eV}$ will not exhibit trap-limited hole transport, similarly, an electron affinity above $3.6 \mathrm{eV}$ will not cause electron trapping to limit electron transport [74]. We also found a similar picture in 10-[4(4,6-diphenyl-1,3,5-triazin-2-yl)phenyl]-9,9-dimethylacridan (DMAC-TRZ) films featuring no deep exponential tail (not shown here) and where the HOMO level is at $5.8 \mathrm{eV}$, consistent with trap-free conditions. Therefore, we assume that the pure Gaussian distribution observed over a wide energy range might be related to the absence of a significant number of extrinsic traps in this material. On the other hand, mCBP has a $\mathrm{HOMO}$ at $6.1 \mathrm{eV}$, which should facilitate extrinsic charge (hole) trapping, correlated with a very pronounced exponential tail observed by TSL (curve 2 in Figure 3b). In the case of mCBP-CN and mCP-CN extrinsic traps are expected, as suggested by HOMO-LUMO level position consideration. However, we hypothesize that significantly stronger molecular dipole moments in mCBP$\mathrm{CN}$ and $\mathrm{mCP}-\mathrm{CN}$ might damp the field variations due to induced dipoles, which give rise predominantly 
to the Gaussian distribution observed in our experiments (cf. curve 3 and 2 in Figure $3 b$ and $4 b$, respectively). These issues definitely require thorough investigation, going beyond the scope of the present study.

Finally, we discuss implication of the 'frequency-to-escape' factor $\langle\mathrm{S}\rangle=2.7 \times 10^{9} \mathrm{~s}^{-1}$ estimated at the maximum of TSL peak (c.f. Figure 2). Since both the TSL and the hopping charge transport process in disordered organic semiconductors are controlled by thermally assisted jumps of relaxed carriers, whose mechanisms have been successfully described in terms by the Miller-Abrahams jump rates [1], it is highly likely that the 'frequency-of-escape frequency' have the same meaning in both cases. For disordered semiconductors, it presents the probability of a charge-carrier hop, i.e. tunnels, when energetic resonance occurs. This probability decreases exponentially with the hopping distance $\rho$ and is usually described in terms of a homogenous lattice gas (also called as the lattice gas model [3]) as

$$
v=v_{0} \exp \left(-2 \rho / \rho_{0}\right)
$$

where $\rho_{0}$ is a wavefunction decay parameter and $v_{0}$ is a frequency factor, which is typically assumed to be equal to the vibrational frequency $\sim 10^{13} \mathrm{~s}^{-1}$. The lattice gas model is based on assumption that the hopping distance can be described as: $\rho=(M / A c \delta)^{1 / 3}$ where $M$ is molecular weight, $A$ is Avogadro's number, $\delta$ is the sample density. The model ignores the distribution of hopping distances as well as the distribution of hopping site energies. The Eq.(8) has been a key relation for describing the dependence of the charge mobility on concentration of charge transporting molecules in molecularly doped polymers [3]. Assuming that the hopping distance $\rho$ in $\mathrm{mCBP}-\mathrm{CN}$ films is equal to the average lattice constant $a=11 \AA$ as estimated above for this material, and that the estimated TSL frequency to escape factor $\langle\mathrm{S}\rangle=2.7 \times 10^{9} \mathrm{~s}^{-1}$ can be described by the lattice gas model, Eq.(8) yields the wavefunction decay parameter $\rho_{0}=2.68 \AA$. This parameter coincides very well with that reported in literature [3], for conventional charge transporting molecules, which is typically within a range of 1-2 $\AA$ and less often approaches $3 \AA$. Thus, the relatively large wavefunction decay parameter implies an enhanced intermolecular electronic coupling, that should promote good transport behavior in this material. This is in line with recent simulation results [70] that predicted a large average coupling in CBP due to the specific shape of its electronic orbitals, which are uniformly distributed over the periphery of the molecule. This facilitates the overlap of states participating in the charge transport and, as a result, leads to a fairly large mobility prefactor $\mu_{0}=0.8 \mathrm{~cm}^{2} \mathrm{~V}^{-1} \mathrm{~s}^{-1}$ in this material.

\section{Conclusions}

We have demonstrated that the DOS distribution in neat OLED host materials can be well determined by low-temperature fractional TSL technique. This is proven by comparison of the TSL results with DOS parameters obtained by computer simulations, carried out in the present paper and from available 
charge transport measurements. We performed comparative TSL studies of two series of OLED host materials possessing different polarity: (i) $\mathrm{CBP}, \mathrm{mCBP}, \mathrm{mCBP}-\mathrm{CN}$, and (i) $\mathrm{mCP}, \mathrm{mCP}-\mathrm{CN}$. Their dipole moments vary from almost zero for non-polar CBP, to 6 Debye in strongly polar mCP-CN. We found that the DOS distribution broadens significantly with increasing molecular dipole moments, which can be well described by the dipolar disorder model, but only for materials belonging to the same series of derivatives which possess similar polarizability of cations and neutral molecules. We have found that the DOS widths of these two material series are offset by a constant amount, with a broader DOS for CPderivatives, compared to CBP-based compounds at equivalent polarity. This was confirmed by simulations that demonstrated a similar trend in the variation of DOS. Larger energetic disorder in CPderivatives is attributed to smaller polarizability of both their neutral and cation states. Therefore, the electrostatic disorder of $\mathrm{CP}$ derivatives is not screened by induction as much as the disorder of CBP derivatives. Additionally, the significant non-polar component of the disorder observed in CP-derivatives is consistent with the theoretical prediction. The dipolar energy disorder component of the DOS, extracted from the TSL experiment, demonstrates similar increase with the magnitude of the molecular dipole moment for all materials considered in this work, implying that the dipolar disorder model is well applicable to such OLED host materials. Such a behavior is expected if the intersite distance is similar in these systems. Finally, we found that a deep exponential tail accompanies Gaussian distribution in CBP and mCBP films. The origin of this effect will be a subject of further investigation of the authors.

\section{Acknowledgements}

The authors acknowledge funding through the EU Marie Skłodowska-Curie ITN TADFlife grant (GA no. 812872). This research was also supported by the European Research Council under the ERC grant agreement no. 835133 (ULTRA-LUX), VW Foundation, and by the National Academy of Science of Ukraine (Project No. VC/205) and NRFU 2020.01/0144. The authors thank Rama Dhali and Anna Painelli from University of Parma for calculation of dipole moments of the materials studied in this paper. D.A. thanks the BMBF grant InterPhase (FKZ 13N13661) and the European Union Horizon 2020 research and innovation program 'Widening materials models' under Grant Agreement No. 646259 (MOSTOPHOS). This research has been supported by the King Abdullah University of Science and Technology (KAUST), via the Competitive Research Grants (CRG) Program. DA acknowledges KAUST for hosting his sabbatical.

\section{References}

[1] H. Bässler, Charge transport in disordered organic photoconductors a Monte Carlo simulation study, Phys. Status Solidi B 175, 15 (1993).

[2] A. Köhler and H. Bässler, Electronic processes in organic semiconductors: An introduction (John Wiley \& Sons, 2015). 
[3] P. M. Borsenberger and D. Weiss, Organic photoreceptors for xerography (CRC Press, 1998).

[4] S. D. Baranovskii, Mott lecture: Description of charge transport in disordered organic semiconductors: Analytical theories and computer simulations, Phys. Status Solidi A 215, 1700676 (2018).

[5] J. Oelerich, D. Huemmer, and S. Baranovskii, How to find out the density of states in disordered organic semiconductors, Phys. Rev. Lett. 108, 226403 (2012).

[6] S. V. Novikov, Far tails of the density of states in amorphous organic semiconductors, J. Chem. Phys. 143, 164510 (2015).

[7] V. Arkhipov, P. Heremans, E. Emelianova, and H. Baessler, Effect of doping on the density-ofstates distribution and carrier hopping in disordered organic semiconductors, Phys. Rev. B 71, 045214 (2005).

[8] S. Van Mensfoort, J. Billen, S. Vulto, R. Janssen, and R. Coehoorn, Electron transport in polyfluorene-based sandwich-type devices: Quantitative analysis of the effects of disorder and electron traps, Phys. Rev. B 80, 033202 (2009).

[9] F. May, B. Baumeier, C. Lennartz, and D. Andrienko, Can lattice models predict the density of states of amorphous organic semiconductors?, Phys. Rev. Lett. 109, 136401 (2012).

[10] O. Tal, Y. Rosenwaks, Y. Preezant, N. Tessler, C. Chan, and A. Kahn, Direct determination of the hole density of states in undoped and doped amorphous organic films with high lateral resolution, Phys. Rev. Lett. 95, 256405 (2005).

[11] K. Celebi, P. Jadhav, K. Milaninia, M. Bora, and M. Baldo, The density of states in thin film copper phthalocyanine measured by Kelvin probe force microscopy, Appl. Phys. Lett. 93, 317 (2008).

[12] M. Pope and C. E. Swenberg, Electronic processes in organic solids, Annu. Rev. Phys. Chem. 35, 613 (1984).

[13] H. Bässler, Exciton and Charge Carrier Transport in Random Organic Solids. In: Richert R., Blumen A. (eds) Disorder Effect on Relaxational Processes (Springe-Verlagr, Berlin Heidelberg New York, 1994, p. 485-507).

[14] F. Schauer, S. Nešpůrek, and H. Valerián, Temperature dependent space-charge-limited currents in amorphous and disordered semiconductors, J. Appl. Phys. 80, 880 (1996).

[15] S. Olthof, S. Mehraeen, S. K. Mohapatra, S. Barlow, V. Coropceanu, J.-L. Brédas, S. R. Marder, and A. Kahn, Ultralow doping in organic semiconductors: evidence of trap filling, Phys. Rev. Lett. 109, 176601 (2012). 
[16] V. Y. Aristov, O. Molodtsova, V. Maslyuk, D. Vyalikh, V. Zhilin, Y. A. Ossipyan, T. Bredow, I. Mertig, and M. Knupfer, Electronic structure of pristine CuPc: Experiment and calculations, Appl. Surf. Sci. 254, 20 (2007).

[17] I. Hulea, H. Brom, A. Houtepen, D. Vanmaekelbergh, J. Kelly, and E. Meulenkamp, Wide energywindow view on the density of states and hole mobility in poly ( $p$-phenylene vinylene), Phys. Rev. Lett. 93, 166601 (2004).

[18] A. Kadashchuk, Y. Skryshevskii, A. Vakhnin, N. Ostapenko, V. Arkhipov, E. Emelianova, and H. Bässler, Thermally stimulated photoluminescence in disordered organic materials, Phys. Rev. B 63, 115205 (2001).

[19] V. Arkhipov, E. Emelianova, A. Kadashchuk, and H. Bässler, Hopping model of thermally stimulated photoluminescence in disordered organic materials, Chem. Phys. 266, 97 (2001).

[20] A. Kadashchuk, A. Vakhnin, Y. Skryshevski, V. Arkhipov, E. Emelianova, and H. Bässler, Thermally stimulated luminescence in $\pi$-conjugated polymers containing fluorene and spirobifluorene units, Chem. Phys. 291, 243 (2003).

[21] A. Kadashchuk, N. Ostapenko, V. Zaika, and S. Nešpurek, Low-temperature thermoluminescence in poly (methyl-phenylsilylene), Chem. Phys. 234, 285 (1998).

[22] A. Kadashchuk, Y. Skryshevski, Y. Piryatinski, A. Vakhnin, E. Emelianova, V. Arkhipov, H. Bässler, and J. Shinar, Thermally stimulated photoluminescence in poly (2, 5-dioctoxy p-phenylene vinylene), J. Appl. Phys. 91, 5016 (2002).

[23] S. A. Patil, U. Scherf, and A. Kadashchuk, New conjugated ladder polymer containing carbazole moieties, Adv. Funct. Mater. 13, 609 (2003).

[24] A. Kadashchuk, S. Schols, A. Vakhnin, J. Genoe, and P. Heremans, Triplet dynamics and charge carrier trapping in triplet-emitter doped conjugated polymers, Chem. Phys. 358, 147 (2009).

[25] A. Kadashchuk, V. I. Arkhipov, C.-H. Kim, J. Shinar, D.-W. Lee, Y.-R. Hong, J.-I. Jin, P. Heremans, and H. Bässler, Localized trions in conjugated polymers, Phys. Rev. B 76, 235205 (2007).

[26] I. Fishchuk, A. Kadashchuk, A. Vakhnin, Y. Korosko, H. Bässler, B. Souharce, and U. Scherf, Transition from trap-controlled to trap-to-trap hopping transport in disordered organic semiconductors, Phys. Rev. B 73, 115210 (2006).

[27] A. Kadashchuk, D. Weiss, P. Borsenberger, S. Nešpurek, N. Ostapenko, and V. Zaika, The origin of thermally stimulated luminescence in neat and molecularly doped charge transport polymer systems, Chem. Phys. 247, 307 (1999). 
[28] A. Kadashchuk, D. Weiss, P. Borsenberger, N. Ostapenko, V. Zaika, and Y. Skryshevski, Effect of extrinsic traps on thermally stimulated luminescence in molecularly doped polymers, Synth. Met. 109, 177 (2000).

[29] P. V. Soroka, A. Y. Vakhnin, Y. A. Skryshevskiy, O. P. Boiko, M. I. Anisimov, Y. L. Slominskiy, V. G. Nazarenko, J. Genoe, and A. Kadashchuk, Charge carrier trapping in highly-ordered lyotropic chromonic liquid crystal films based on ionic perylene diimide derivatives, Eur. Phys. J.-Appl. Phys., 68, 30201 (2014).

[30] A. Fakharuddin et al., Reduced Efficiency Roll-Off and Improved Stability of Mixed 2D/3D Perovskite Light Emitting Diodes by Balancing Charge Injection, Adv. Funct. Mater. 29, 1904101 (2019).

[31] I. Tale, Trap spectroscopy by the fractional glow technique, Phys. Status Solidi A 66, 65 (1981).

[32] H. Gobrecht and D. Hofmann, Spectroscopy of traps by fractional glow technique, J. Phys. Chem. Solids, 27, 509 (1966).

[33] Y. Gorohovatsky and H. Bordovsky, Thermally activational current spectroscopy of highresistance semiconductors and dielectrics, Izd. Nauka, Moscow (1991).

[34] W. L. Jorgensen and J. Tirado-Rives, Potential energy functions for atomic-level simulations of water and organic and biomolecular systems, Proc. Natl. Acad. Sci., 102, 6665 (2005).

[35] W. L. Jorgensen and J. Tirado-Rives, The OPLS [optimized potentials for liquid simulations] potential functions for proteins, energy minimizations for crystals of cyclic peptides and crambin, J. Am. Chem. Soc. 110, 1657 (1988).

[36] W. L. Jorgensen, D. S. Maxwell, and J. Tirado-Rives, Development and testing of the OPLS allatom force field on conformational energetics and properties of organic liquids, J. Am. Chem. Soc. 118, 11225 (1996).

[37] C. M. Breneman and K. B. Wiberg, Determining atom-centered monopoles from molecular electrostatic potentials. The need for high sampling density in formamide conformational analysis, J. Comput. Chem. 11, 361 (1990).

[38] C. Poelking, E. Cho, A. Malafeev, V. Ivanov, K. Kremer, C. Risko, J.-L. Brédas, and D. Andrienko, Characterization of charge-carrier transport in semicrystalline polymers: electronic couplings, site energies, and charge-carrier dynamics in poly (bithiophene-alt-thienothiophene)[PBTTT], J. Phys. Chem. C 117, 1633 (2013).

[39] G. Bussi, D. Donadio, and M. Parrinello, Canonical sampling through velocity rescaling, J. Chem. Phys., 126, 014101 (2007). 
[40] H. J. Berendsen, J. v. Postma, W. F. van Gunsteren, A. DiNola, and J. R. Haak, Molecular dynamics with coupling to an external bath, J. Chem. Phys., 81, 3684 (1984).

[41] B. Hess, C. Kutzner, D. Van Der Spoel, and E. Lindahl, GROMACS 4: algorithms for highly efficient, load-balanced, and scalable molecular simulation, J. Chem. Theory Comput., 4, 435 (2008).

[42] S. Pronk et al., GROMACS 4.5: a high-throughput and highly parallel open source molecular simulation toolkit, Bioinformatics 29, 845 (2013).

[43] A. Stone, The theory of intermolecular forces (Clarendon Press, Oxford, 1997).

[44] G. D’Avino, L. Muccioli, F. Castet, C. Poelking, D. Andrienko, Z. G. Soos, J. Cornil, and D. Beljonne, Electrostatic phenomena in organic semiconductors: fundamentals and implications for photovoltaics, J. Phys.: Condens. Matter., 28, 433002 (2016).

[45] C. Poelking and D. Andrienko, Long-range embedding of molecular ions and excitations in a polarizable molecular environment, J. Chem. Theory Comput., 12, 4516 (2016).

[46] B. T. Thole, Molecular polarizabilities calculated with a modified dipole interaction, Chem. Phys., 59, 341 (1981).

[47] P. T. Van Duijnen and M. Swart, Molecular and atomic polarizabilities: Thole's model revisited, J. Phys. Chem. A, 102, 2399 (1998).

[48] A. J. Stone, Distributed multipole analysis: Stability for large basis sets, J. Chem. Theory Comput., 1,1128 (2005).

[49] V. Rühle, A. Lukyanov, F. May, M. Schrader, T. Vehoff, J. Kirkpatrick, B. r. Baumeier, and D. Andrienko, Microscopic simulations of charge transport in disordered organic semiconductors, J. Chem. Theory Comput., 7, 3335 (2011).

[50] E. W. Forsythe, D. C. Morton, C. W. Tang, and Y. Gao, Trap states of tris-8-(hydroxyquinoline) aluminum and naphthyl-substituted benzidine derivative using thermally stimulated luminescence, Appl. Phys. Lett. 73, 1457 (1998).

[51] J. Simmons and G. Taylor, High-field isothermal currents and thermally stimulated currents in insulators having discrete trapping levels, Phys. Rev. B 5, 1619 (1972).

[52] G. G. Malliaras, Y. Shen, D. H. Dunlap, H. Murata, and Z. H. Kafafi, Nondispersive electron transport in Alq 3, Appl. Phys. Lett. 79, 2582 (2001).

[53] H. Bässler, Localized states and electronic transport in single component organic solids with diagonal disorder, Phys. Stat. Sol. B 107, 9 (1981). 
[54] R. Eiermann, W. Hofberger, and H. Bässler, Localized valence states in the forbidden gap of noncrystalline tetracene, J. Non·Cryst. Solids 28, 415 (1978).

[55] B. Movaghar, B. Ries, and M. Grünewald, Diffusion and relaxation of energy in disordered systems: Departure from mean-field theories, Phys. Rev. B 34, 5574 (1986).

[56] M. Era, K. Mori, and N. Mototsu, Analysis of Hole Mobility of Fluorene Derivative Films Based on the Disorder Model and Relationship between Disorder Free Mobility and Reorganization Energy in the Marcus Theory. In: Organic Light Emitting Materials and Devices XVII, Vol. 8829 (International Society for Optics and Photonics, 2013), p. 88290N.

[57] S. Athanasopoulos, S. T. Hoffmann, H. Bässler, A. Köhler, and D. Beljonne, To hop or not to hop? Understanding the temperature dependence of spectral diffusion in organic semiconductors, J. Phys. Chem. Lett. 4, 1694 (2013).

[58] A. Dieckmann, H. Bässler, and P. Borsenberger, An assessment of the role of dipoles on the density-of-states function of disordered molecular solids, J. Chem. Phys., 99, 8136 (1993).

[59] P. Borsenberger and H. Bässler, Concerning the role of dipolar disorder on charge transport in molecularly doped polymers, J. Chem. Phys., 95, 5327 (1991).

[60] P. Borsenberger, in Electrical and Related Properties of Organic Solids (Springer, 1997), pp. 25.

[61] S. Novikov and A. Vannikov, Hopping charge transport in disordered organic materials: where is the disorder?, J. Phys. Chem. C 113, 2532 (2009).

[62] R. H. Young, Dipolar lattice model of disorder in random media analytical evaluation of the gaussian disorder model, Philos. Mag. B 72, 435 (1995).

[63] C. Madigan and V. Bulović, Charge carrier energy disorder in polar amorphous organic thin films, Phys. Rev. Lett. 97, 216402 (2006).

[64] P. Borsenberger, M. Detty, and E. Magin, Electron transport in vapor deposited molecular glasses, Phys. Stat. Sol. B 185, 465 (1994).

[65] P. M. Borsenberger and J. J. Fitzgerald, Effects of the dipole moment on charge transport in disordered molecular solids, J. Phys. Chem. 97, 4815 (1993).

[66] V. Mimaite, J. V. Grazulevicius, R. Laurinaviciute, D. Volyniuk, V. Jankauskas, and G. Sini, Can hydrogen bonds improve the hole-mobility in amorphous organic semiconductors? Experimental and theoretical insights, J. Mater. Chem. C 3, 11660 (2015).

[67] P. Borsenberger, E. Magin, M. O'regan, and J. Sinicropi, The role of dipole moments on hole transport in triphenylamine-doped polymers, J. Polym. Sci., Part B: Polym. Phys. 34, 317 (1996). 
[68] P. Borsenberger and M. O'Regan, The role of dipole moments on hole transport in triphenylamine doped poly (styrene), Chem. Phys. 200, 257 (1995).

[69] R. H. Young and J. J. Fitzgerald, Effect of polar additives on charge transport in a molecularly doped polymer: Survey of various additives, J. Chem. Phys 102, 2209 (1995).

[70] N. B. Kotadiya, A. Mondal, S. Xiong, P. W. Blom, D. Andrienko, and G. J. A. Wetzelaer, Rigorous characterization and predictive modeling of hole transport in amorphous organic semiconductors, Adv. Electron. Mater. 4, 1800366 (2018).

[71] P. K. Nayak and N. Periasamy, Calculation of ionization potential of amorphous organic thin-films using solvation model and DFT, Org. Electron. 10, 532 (2009).

[72] S. Van Mensfoort, R. De Vries, V. Shabro, H. Loebl, R. Janssen, and R. Coehoorn, Electron transport in the organic small-molecule material BAlq-the role of correlated disorder and traps, Org. Electron. 11, 1408 (2010).

[73] S. Van Mensfoort and R. Coehoorn, Effect of Gaussian disorder on the voltage dependence of the current density in sandwich-type devices based on organic semiconductors, Phys. Rev. B 78, 085207 (2008).

[74] N. B. Kotadiya, A. Mondal, P. W. Blom, D. Andrienko, and G.-J. A. Wetzelaer, A window to trapfree charge transport in organic semiconducting thin films, Nature materials 18, 1182 (2019).

[75] A. Mondal, L. Paterson, J. Cho, K-H. Lin, B. van der Zee, G. Wetzelaer, A. Stankevych, A. Vakhnin, J. Kim, A. Kadashchuk, P.W.M Blom, F. May and D. Andrienko, Physical properties of OLED host materials, (submitted to Chemical Physics Review, 2021).

[76] S.T. Hoffmann, F. Jaiser, A. Hayer, H. Bässler, T. Unger, S. Athanasopoulos, D. Neher, and A. Köhler, How do disorder, reorganization, and localization influence the hole mobility in conjugated copolymers?. J. Am. Chem. Soc., 135, 1772 (2013). 